to appear in Physical Review Letters

IITAP-96-12-01

December 1996

Extended version: March 1997

hep-th/9612147

\title{
Effective Regge QCD
}

\author{
Victor T. Kim ${ }^{\S \dagger}$ and Grigorii B. Pivovarov ${ }^{\S \ddagger}$ \\ $\S$ : International Institute of Theoretical and Applied Physics, \\ Iowa State University, Ames, Iowa 50011-3022, USA \\ $\dagger$ : St.Petersburg Nuclear Physics Institute, 188350 Gatchina, Russia \\ ‡: Institute for Nuclear Research, 117312 Moscow, Russia
}

\begin{abstract}
A new framework for a high energy limit of quantum gauge field theories is introduced. Its potency is illustrated on a new derivation of the reggeization of the gluon.
\end{abstract}

PACS number(s): 11.10.Hi, 11.10.Ji, 11.55.Jy, 12.38.Cy

\footnotetext{
${ }^{1}$ permanent address; e-mail: kim@pnpi.spb.ru

2 permanent address; e-mail: gbpivo@ms2.inr.ac.ru
} 
The concept of effective field theory (for a review, see [1]) is an important means to obtain qualitative understanding of quantum field theories. It is also an important calculational tool.

Effective field theory provides a mechanism of unification - the plethora of its fields is consolidated by this mechanism into a more compact set of fields of the underlying theory which implements a symmetry richer than the one of the effective theory. A well known example is the connection between the standard model and the grand unification models [2]. Another example is nonrelativistic QED [3, 4], where a single fermion field breaks down into two independent fields of fermions and antifermions.

The calculational advantage is obtained via the usage of the renormalization group ( $\mathrm{RG}$ ) invariance [5]. It requires the predictions of the effective theory to be independent of the particular way the fields of the underlying theory were broken into the fields of the effective theory. Solving the RG equations is the most effective way to make a resummation of the perturbative expansion for the underlying theory taken in the corresponding "leading logarithm approximation" (for the notion of the leading logarithms see, for example, [6]).

The necessity to develop an effective theory for Regge limit of gauge theories is well acknowledged [7-10]. It is more pressing now as the energy reached in hadron collisions provides data (see, e.g., [11]) whose understanding requires [12] an account of the resummation of the leading energy logarithms for QCD. For known results on the resummation see [13, 14. Attempts of such an account are available [15].

Here we present a new approach which is, in our opinion, the most straightforward realization of the effective field theory concept for the Regge limit. It allows one to prove the reggeization of the gluon [14, 16] - a property which is, at the moment, a well-tested conjecture.

Consider a Green's function of a field theory,

$$
G\left(x_{1}, \ldots, x_{n}\right)=<T \Phi\left(x_{1}\right) \ldots \Phi\left(x_{n}\right)>,
$$

where any $x_{n}$ comprises all the variables labelling the field $\Phi$ (in particular, the space-time coordinates or the momenta if one chooses the momentum representation). Consider next the Green's function of the boosted fields,

$$
G_{\lambda}\left(x_{1}, \ldots, x_{n} ; y_{1}, \ldots, y_{m}\right)=<T \Phi_{\lambda}\left(x_{1}\right) \ldots \Phi_{\lambda}\left(x_{n}\right) \Phi_{1 / \lambda}\left(y_{1}\right) \ldots \Phi_{1 / \lambda}\left(y_{m}\right)>,
$$

where $\Phi_{\lambda}(x)=B^{-1}(\lambda) \Phi(B(\lambda) x)$ is the Lorentz boost of the field $\Phi$ along a $z$-axis, parameterized by the exponential of the rapidity of the boost, $\lambda=\sqrt{(B(\lambda) p)_{+} /(B(\lambda) p)_{-}}$, for a four-vector $p\left(p_{ \pm}=p_{0} \pm p_{z}\right.$ are the light-cone components of $\left.p\right)$. The Regge limit is then the one of infinite rapidity, i.e., $\lambda \rightarrow \infty$. The effective Regge theory is to approximate the boosted Green's functions of Eq. (1) at large $\lambda$.

Intuitively, the collection of the fields labeled by $x_{i}$ in Eq. (1) represents the excitations moving fast rightwards in the $z$-direction, while the $y$-fields represent the fast left-movers. 
The $\lambda$ then scales as the invariant energy of the relative motion of the left- and rightmovers.

The momenta of the fields in the right hand side (rhs) of Eq. (1) satisfy, for large $\lambda$, either

$$
\left(\left|p_{+}\right|>\mu_{1},\left|p_{-}\right|<\mu_{2}\right) \Leftrightarrow\left(p \in \Omega_{R}\right)
$$

for the right-movers, or

$$
\left(\left|p_{+}\right|<\mu_{2},\left|p_{-}\right|>\mu_{1}\right) \Leftrightarrow\left(p \in \Omega_{L}\right)
$$

for the left-movers. The $\mu_{i}$ are arbitrary scales. The only requirement we need on them is $\Omega_{R} \cap \Omega_{L}=\emptyset$. The RG invariance requires that any physical prediction be independent of their values.

Instead of the single field $\Phi$ of the underlying theory, the effective theory has two independent fields,

$$
\left(R_{1 / \lambda}(x)=\Phi(x)\right)_{\left.\right|_{p \in \Omega_{R}}},\left(L_{\lambda}(x)=\Phi(x)\right)_{\left.\right|_{p \in \Omega_{L}}} .
$$

The $p$ above is supposed to belong to $x$. The $\lambda$-subscript denotes the boost transformation, as in Eq. (1).

With Eq. (4), the boosted Green's function of Eq. (1) is

$$
G_{\lambda}\left(x_{1}, \ldots, x_{n} ; y_{1}, \ldots, y_{m}\right)=<T R\left(x_{1}\right) \ldots R\left(x_{n}\right) L\left(y_{1}\right) \ldots L\left(y_{m}\right)>.
$$

The effective action for the fields $R, L$ is defined by

$$
\exp \left(i S_{e f f}(R, L, \lambda)\right)=\int \prod_{p \notin\left(\Omega_{R} \cup \Omega_{L}\right)} d \Phi(x) \exp (i S(\Phi)) .
$$

Where the rhs is expressed through $R, L$ by Eq. (4).

If the boosted Green's functions of Eq. (1) are finite in the limit $\lambda \rightarrow \infty$ modulo logarithms of $\lambda$, i.e., logarithms of the invariant energy for the relative motion of the leftand right-movers, then the effective action of Eq. (6) should have a finite limit,

$$
S_{e f f}(R, L)=\lim _{\lambda \rightarrow \infty} S_{e f f}(R, L, \lambda) .
$$

In that case, the only dependence on $\lambda$ left in the effective theory enters through the scales $\mu_{i}$ of Eqs. (2),(3) which cut the effective theory since after been boosted the scales get a $\lambda$-dependence. This removes the cuts from the effective theory in the limit $\lambda \rightarrow \infty$. In this way, to study the energy dependence in the Regge limit means to study the infrared and ultraviolet divergences of the effective theory.

Note that the cuts involve only the longitudinal directions. Thus we need to study only the divergences of a 2-dimensional (2D) field theory at fixed values of the transverse 
coordinates (momenta). By transverse coordinates we mean all the variables labelling the $R$ and $L$ fields but the light-cone coordinates $x_{ \pm}$. The coupling "constants" of this $2 \mathrm{D}$ theory depend on the transverse coordinates. The RG equations for the coupling constants are an integral equations in the space of the transverse coordinates. In the case of QCD, these equations contain the same information as the BFKL equation [14.

To check this, consider the gluodynamics as the underlying theory, i.e.,

$$
S_{\text {glue }}(A)=-\frac{1}{4}\left(F_{\mu \nu}^{a}\right)^{2}
$$

with $A$ denoting the color octet of vector gluon fields, $F_{\mu \nu}^{a}=\partial_{\mu} A_{\nu}^{a}-\partial_{\nu} A_{\mu}^{a}-g C_{a b c} A_{\mu}^{b} A_{\nu}^{c}$ and $g$ the gauge coupling. The effective Regge gluodynamics is then a theory of two color octets of vector fields $R$ and $L$. With Eq. (6), its action up to terms of order $g^{2}$ is

$$
S_{\text {eff }}(R, L)=S_{\text {glue }}(R)+S_{\text {glue }}(L)+S_{\text {int }}(R, L),
$$

where $S_{\text {int }}$ is an action of interaction between the right- and left-boosted gluons, bilinear in $R$ and $L$.

Note that the self-interaction of both right- and left-movers mimics the self-interaction of the underlying field. This is a consequence of the Lorentz invariance of the underlying theory.

The $S_{i n t}$-term comes from the diagrams in Fig. 1. Infinitely boosting the fields according with the labels on the external legs of the diagrams from Fig. 1, one obtains a finite expression for $S_{\text {int }}$. The finiteness is a nontrivial outcome of a cancellation of infinities between the contributions of Fig. 1(b) and Fig. 1(c).

Intermediate steps of the following calculation may depend on the gauge. In these cases Feynman gauge is implied.

To specify the form of $S_{\text {int }}$ from Eq. (9), we need the following objects:

$$
\left(\mathcal{N}^{R}\right)_{m}\left(x_{\perp}\right)=\int d x_{-} d y_{-} \partial_{i} R_{+}^{a}\left(x_{-}, x_{\perp}\right) D\left(x_{-}-y_{-}\right) \partial_{i} R_{+}^{b}\left(y_{-}, x_{\perp}\right) C_{m b a},
$$

where $R_{\mu}^{a}$ is the gluon right-movers field taken on a light-front $x_{+}=0, \partial_{i}$ are the derivatives over transverse coordinates (summation over $i=1,2$ is implied), and $D$ is

$$
\begin{gathered}
D(x)=\int \frac{d k}{2 \pi} \frac{e^{i k x}}{i k} \\
\left(\mathcal{M}^{R}\right)_{m}\left(x_{\perp}\right)=\int d x_{-} d y_{-} \partial_{i} \partial_{i}\left(R_{+}^{a}\left(x_{-}, x_{\perp}\right) D\left(x_{-}-y_{-}\right) R_{+}^{b}\left(y_{-}, x_{\perp}\right)\right) C_{m b a}, \\
\left(\mathcal{A}_{1}^{R}\right)_{m}\left(x_{\perp}\right)=\int d x_{-}\left(\partial^{-} R_{+}^{a}\left(x_{-}, x_{\perp}\right) R_{-}^{b}\left(x_{-}, x_{\perp}\right)+\partial^{-} R_{-}^{a}\left(x_{-}, x_{\perp}\right) R_{+}^{b}\left(x_{-}, x_{\perp}\right)+\right. \\
\left.+R_{+}^{a}\left(x_{-}, x_{\perp}\right) \partial^{+} R_{+}^{b}\left(x_{-}, x_{\perp}\right)-\partial^{+} R_{+}^{a}\left(x_{-}, x_{\perp}\right) R_{+}^{b}\left(x_{-}, x_{\perp}\right)\right) C_{m b a},
\end{gathered}
$$




$$
\begin{gathered}
\left(\mathcal{A}_{2}^{R}\right)_{m}\left(x_{\perp}\right)=\int d x_{-}\left(\partial^{i} R_{i}^{a}\left(x_{-}, x_{\perp}\right) R_{+}^{b}\left(x_{-}, x_{\perp}\right)-R_{+}^{a}\left(x_{-}, x_{\perp}\right) \partial^{i} R_{i}^{b}\left(x_{-}, x_{\perp}\right)\right) C_{m b a} \\
\left(\mathcal{A}_{3}^{R}\right)_{m}\left(x_{\perp}\right)=\int d x_{-}\left(R_{i}^{a}\left(x_{-}, x_{\perp}\right) \partial^{i} R_{+}^{b}\left(x_{-}, x_{\perp}\right)-\partial^{i} R_{+}^{a}\left(x_{-}, x_{\perp}\right) R_{i}^{b}\left(x_{-}, x_{\perp}\right)\right) C_{m b a} \\
\left(\mathcal{A}_{4}^{R}\right)_{m}\left(x_{\perp}\right)=\int d x_{-} \partial^{-} R_{i}^{a}\left(x_{-}, x_{\perp}\right) R_{i}^{b}\left(x_{-}, x_{\perp}\right) C_{m b a} .
\end{gathered}
$$

The derivatives in the above equations act only on their nearest right neighbors. For further convenience, the following Fourier transformations of linear combinations of the above operators are intoduced:

$$
\begin{array}{r}
\tilde{\mathcal{N}}_{m}^{R}\left(q_{\perp}\right)=\frac{\pi}{2} \int \frac{d^{2} x_{\perp}}{(2 \pi)^{2}} e^{-i q_{\perp} x_{\perp}}\left(\frac{1}{2}\left(\mathcal{N}^{R}\right)_{m}\left(x_{\perp}\right)+\frac{1}{4}\left(\mathcal{M}^{R}\right)_{m}\left(x_{\perp}\right)-\frac{1}{2}\left(\mathcal{A}_{1}^{R}\right)_{m}\left(x_{\perp}\right)-\right. \\
\left.-\frac{1}{2}\left(\mathcal{A}_{2}^{R}\right)_{m}\left(x_{\perp}\right)-2\left(\mathcal{A}_{3}^{R}\right)_{m}\left(x_{\perp}\right)+4\left(\mathcal{A}_{4}^{R}\right)_{m}\left(x_{\perp}\right)\right), \\
\tilde{\mathcal{M}}_{m}^{R}\left(q_{\perp}\right)=\frac{\pi}{2} \int \frac{d^{2} x_{\perp}}{(2 \pi)^{2}} e^{-i q_{\perp} x_{\perp}}\left(\frac{1}{2}\left(\mathcal{N}^{R}\right)_{m}\left(x_{\perp}\right)-\frac{1}{4}\left(\mathcal{M}^{R}\right)_{m}\left(x_{\perp}\right)-\frac{1}{2}\left(\mathcal{A}_{1}^{R}\right)_{m}\left(x_{\perp}\right)-\right. \\
\left.\quad-\frac{1}{2}\left(\mathcal{A}_{2}^{R}\right)_{m}\left(x_{\perp}\right)-2\left(\mathcal{A}_{3}^{R}\right)_{m}\left(x_{\perp}\right)+4\left(\mathcal{A}_{4}^{R}\right)_{m}\left(x_{\perp}\right)\right), \\
\tilde{\mathcal{J}}_{m}^{R}\left(q_{\perp}\right)=\frac{\pi}{2} \int \frac{d^{2} x_{\perp}}{(2 \pi)^{2}} e^{-i q_{\perp} x_{\perp}}\left(\left(\mathcal{A}_{1}^{R}\right)_{m}\left(x_{\perp}\right)+\left(\mathcal{A}_{2}^{R}\right)_{m}\left(x_{\perp}\right)+2\left(\mathcal{A}_{3}^{R}\right)_{m}\left(x_{\perp}\right)-4\left(\mathcal{A}_{4}^{R}\right)_{m}\left(x_{\perp}\right)\right), \\
\tilde{\mathcal{L}}_{m}^{R}\left(q_{\perp}\right)=\frac{\pi}{2} \int \frac{d^{2} x_{\perp}}{(2 \pi)^{2}} e^{-i q_{\perp} x_{\perp}}\left(\left(\mathcal{A}_{1}^{R}\right)_{m}\left(x_{\perp}\right)+\left(\mathcal{A}_{2}^{R}\right)_{m}\left(x_{\perp}\right)+4\left(\mathcal{A}_{3}^{R}\right)_{m}\left(x_{\perp}\right)-4\left(\mathcal{A}_{4}^{R}\right)_{m}\left(x_{\perp}\right)\right) .
\end{array}
$$

We also need the same set of objects for the left-movers; the definitions may be contrived from Eqs. (10)-(20) by the substitutions $R \rightarrow L,+\rightarrow-,-\rightarrow+$ (the last two substitutions act on the longitudinal Lorentz indices).

$S_{\text {int }}$ is expressed in terms of these objects as

$$
\begin{array}{r}
S_{i n t}(R, L)=\int d^{2} q_{\perp}\left(G _ { 0 } ( q _ { \perp } ) \left(\tilde{\mathcal{J}}_{m}^{R}\left(q_{\perp}\right) \tilde{\mathcal{J}}_{m}^{L}\left(-q_{\perp}\right)-\tilde{\mathcal{M}}_{m}^{R}\left(q_{\perp}\right) \tilde{\mathcal{M}}_{m}^{L}\left(-q_{\perp}\right)-\right.\right. \\
\left.-\tilde{\mathcal{M}}_{m}^{R}\left(q_{\perp}\right) \tilde{\mathcal{L}}_{m}^{L}\left(-q_{\perp}\right)-\tilde{\mathcal{L}}_{m}^{R}\left(q_{\perp}\right) \tilde{\mathcal{M}}_{m}^{L}\left(-q_{\perp}\right)\right)+ \\
+G_{1}\left(q_{\perp}\right)\left(\tilde{\mathcal{N}}_{m}^{R}\left(q_{\perp}\right) \tilde{\mathcal{L}}_{m}^{L}\left(-q_{\perp}\right)+\tilde{\mathcal{L}}_{m}^{R}\left(q_{\perp}\right) \tilde{\mathcal{N}}_{m}^{L}\left(-q_{\perp}\right)\right)+ \\
\left.+G_{2}\left(q_{\perp}\right) \tilde{\mathcal{N}}_{m}^{R}\left(q_{\perp}\right) \tilde{\mathcal{N}}_{m}^{L}\left(-q_{\perp}\right)\right)
\end{array}
$$

where summation over colour indices $m$ is implied.

The "coupling constants" $G_{k=0,1,2}\left(q_{\perp}\right)$ depend on the transverse momentum transferred from the right-movers to the left-movers:

$$
G_{k}\left(q_{\perp}\right)=\frac{g^{2}}{-q_{\perp}^{2}} .
$$


We need to distinguish between them because they couple different number of $\mathcal{N}$-operators.

The first $\mathcal{J}^{R} \mathcal{J}^{L}$-term of Eq. (21) is the contribution of Fig. 1(a) originating from the interaction between the left- and right-movers via exchange of a gluon which momentum has $p_{ \pm}<\mu_{1}$.

It is important to note the following: right-movers interact with the left-movers only by their values at the light-front $x_{+}=0$, while the left movers interact only by their values on the perpendicular light-front $x_{-}=0$. It is also important that $\mathcal{N}, \mathcal{M}$ are nonlocal on the corresponding light-fronts, while $\mathcal{A}_{i}$ are local.

We stress that Eq. (21) is only the leading order contribution to the expansion over the coupling constant of the action of interaction between the left- and right-boosted gluons. It remains an important unsolved problem to establish the general form of this action of interaction in higher orders of and beyond the perturbation theory.

Next we consider the divergences of the one-loop Feynman diagrams generated by $S_{\text {eff }}$ of Eq. (9). We need to consider the divergences in the longitudinal integrations arising as the cuts on the longitudinal momenta from Eq. (2) for the $R$-field and from Eq. (3) for the $L$-field are removed. That should be done at fixed transverse coordinates.

It turns out that there are only two divergent diagrams in the one-loop approximation (Fig. 2). They are logarithmically divergent in the infrared because of the singularity $1 / i k$ in the rhs of Eq. (11). As it can be seen in the diagrams, the problem is factorized: calculation of the divergences of Fig. 2(a) does not involve the $L$-fields, while calculation of Fig. 2(b) does not involve the $R$-fields. Thus, it suffices to study the infrared divergences generated by the self-interaction of the $R$-fields in the operators $\tilde{\mathcal{N}}_{m}^{R}, \tilde{\mathcal{M}}_{m}^{R}$ of Eqs. (17),(18). The left sector gives the same divergences.

It is a remarkable fact that these infrared divergences may be absorbed in a multiplicative renormalization of the operators $\tilde{\mathcal{N}}_{m}^{R, L}$. Namely, a simple calculation shows that

$$
\text { d.p. }\left\langle\tilde{\mathcal{N}}_{m}^{R, L}\left(q_{\perp}\right)\right\rangle=\log (\lambda) \alpha\left(q_{\perp}\right) \tilde{\mathcal{N}}_{m}^{R, L}\left(q_{\perp}\right),
$$

where d.p. means divergent part and the angle brackets denote the correction of the $\mathcal{N}_{m}^{R}$ $\left(\mathcal{N}_{m}^{L}\right)$ for the self-interaction of the $R$-fields ( $L$-fields), and $\lambda$ is the exponential of the rapidity of the boost. The rest of the operators $(\mathcal{M}, \mathcal{J}, \mathcal{L})$ participating in Eq. (21) are finite in the one-loop approximation. We should note that Eq. (23) was obtained with the dimensional regularization of the integration over transverse momentum involved (in particular, integrals like $\int d l_{\perp} / l_{\perp}^{2}$ was set to zero).

The $\alpha\left(q_{\perp}\right)$ of Eq. (23) determines the leading contribution to the renormalization "constant" of the operators $\tilde{\mathcal{N}}_{m}^{R, L}\left(q_{\perp}\right)$. It turns out to be

$$
\alpha\left(q_{\perp}\right)=3 \alpha_{S} q_{\perp}^{2} \int \frac{d^{2} k_{\perp}}{(2 \pi)^{2} k_{\perp}^{2}(k-q)_{\perp}^{2}}
$$

coinciding with the known Regge trajectory of the reggeized gluon [14, 16]. 
It follows from Eq. (23) and the one-loop finiteness of the operators $\mathcal{M}, \mathcal{J}, \mathcal{L}$ that all one-loop divergences generated by the effective action of Eq. (21) may be removed by a renormalization of the coupling constants, $G_{k}\left(q_{\perp}\right) \rightarrow G_{k, R}\left(q_{\perp}\right)=Z_{k}\left(q_{\perp}\right) G_{k}\left(q_{\perp}\right)$. The renormalization constants $Z_{k}\left(q_{\perp}\right), k=0,1,2$, are

$$
Z_{k}\left(q_{\perp}\right)=1-k \log (\lambda) \alpha\left(q_{\perp}\right)
$$

in the one-loop approximation.

This allows one to apply the standard RG considerations (see, e.g., a text-book [17]). In particular, running couplings $\bar{G}_{k}\left(q_{\perp}\right)$ may be defined as functions of the logarithm of the boosted cut $\mu_{1} / \lambda$. The standard procedure yields the following RG equation for the running couplings:

$$
\frac{\partial \bar{G}_{k}\left(q_{\perp}\right)}{\partial \log (\lambda)}=-\frac{1}{Z_{k}\left(q_{\perp}\right)} \frac{\partial Z_{k}\left(q_{\perp}\right)}{\partial \log (\lambda)} \bar{G}_{k}\left(q_{\perp}\right) \approx k \alpha\left(q_{\perp}\right) \bar{G}_{k}\left(q_{\perp}\right),
$$

which solution is

$$
\bar{G}_{k}\left(q_{\perp}\right)=\lambda^{k \alpha\left(q_{\perp}\right)} \frac{g^{2}}{-q_{\perp}^{2}}
$$

(this takes into account that boundary values of $\bar{G}_{k}\left(q_{\perp}\right)$ at $\lambda=1$ are given by Eq. (22)).

To conclude, we formulated an effective Regge gauge field theory and recognized the problem of resummation of energy logarithms for gauge theories as a problem of infrared renormalization of some nonlocal operators in a 2D field theory. The trajectory of the reggeized gluon was rederived in this way. In a forthcoming article [18] we shall show that the same renormalization of the nonlocal operators contains, in the two-loop approximation, the BFKL equation.

We thank I.Ya. Aref'eva, I.I. Balitsky, B.I. Ermolaev, L.D. Faddeev, I.F. Ginzburg, V.A. Kuzmin, E.M. Levin, L.N. Lipatov, V.A. Matveev, N.N. Nikolaev, A. Petridis, J.P. Vary, and A.A. Vorobyov for stimulating discussions. We are grateful to the Fermilab Theory Division for warm hospitality. V.T.K. is thankful to the Aspen Center for Physics

for its hospitality. G.B.P. wishes to thank L. McLerran and R. Venugopalan for fruitful discussions, and also the Institute for the Nuclear Theory of the University of Washington, Seattle and the Nordita, Copenhagen for their kind hospitality. This work was supported in part by the Russian Foundation for Basic Research, grants No. 96-02-16717 and 9602-18897.

\section{References}

[1] J. Polchinski, in Proc. TASI-92, Boulder, CO, Jun. 3-28, 1992, edited by J. Harvey and J. Polchinski, (World Scientific, 1993), hep-ph/9210046; 
H. Georgi, Ann. Rev. Nuc. Part. Sci. 43, 209 (1994);

D.B. Kaplan, Lectures presented at the 7th Summer School in Nucl. Physics: "Symmetries", Jun. 19-30, 1995, Seattle, WA UW/PT 95-05, nucl-th/9506035;

A.V. Manohar, Lectures presented at the Int. Winter School of Nucl. and Part. Physics: Pert. and Nonpert. Aspects of Quantum Field Theory, Schladming, Austria, Mar. 2-9 1996, UCSD/PTH 96-04, hep-ph/9606222

[2] H. Georgi, Weak Interactions and Modern Particle Theory (Benjamin, 1984)

[3] W.E. Caswell and G.P. Lepage, Phys. Lett. 167 B, 437 (1986)

[4] G.B. Pivovarov, in Proc. of Int. Seminar "Quarks-94", Vladimir, Russia, May, 1994, edited by D.Yu. Grigoriev, V.A. Matveev, and V.A.Rubakov (World Scientific, 1994)

[5] K.G. Wilson and J. Kogut, Phys. Rep. C 12, 75 (1974)

[6] L.V. Gribov, E.M. Levin and M.G. Ryskin, Phys. Rep. C 100, 1 (1983)

[7] H. Verlinde and E. Verlinde, Report PUPT-1319, Princeton, 1993, hep-th/9302104

[8] S.J. Rey, in Proc. of 5th Blois Workshop on Elastic and Diffractive Scattering, Providence, RI, June 8-12, 1993, edited by H.M. Fried, K. Kang, and C.-I. Tan (World Scientific, 1994), hep-ph/9308332

[9] I.Ya.Aref'eva, Phys. Lett. 325 B, 171 (1994)

[10] L.N.Lipatov, Nucl.Phys. B 452, 369 (1995)

[11] CDF Collaboration, F. Abe et al., Phys. Rev. Lett. 70, 1376 (1993);

T.Devlin, FERMILAB-CONF-96-350-E (1996), presented at the XXVIII ICHEP96 Warsaw, Poland, Jul. 25-31, 1996;

A.A. Bhatti, FERMILAB-CONF-96-352-E, presented at the DPF96 Meeting, Minneapolis, MN, August 10-15, 1996

[12] W. Giele, talk presented at the CTEQ Symposium, Confronting QCD with Experiment: Puzzles and Challenges, Fermilab, IL, Nov. 7-9, 1996

[13] L.N.Lipatov, DESY-96-132 (1996), hep-ph/9610276 (to appear in Phys. Rep. C)

[14] L.N.Lipatov, Yad. Fiz. 23, 642 (1976) [Sov. J. Nucl. Phys. 23, 338 (1976)];

E.A.Kuraev, L.N.Lipatov and V.S.Fadin, Zh. Eksp. Teor. Fiz. 71, 840 (1976) [Sov. JETP 44, 443 (1976)]; 72, 377 (1977) [45, 199 (1977)];

Ya.Ya.Balitskii and L.N.Lipatov, Yad. Fiz. 28, 1597 (1978) [Sov. J. Nucl. Phys. 28, $822(1978)]$;

L.N.Lipatov, Zh. Eksp. Teor. Fiz. 90, 1536 (1986) [Sov. JETP 63, 904 (1986)] 
[15] A.H. Mueller and H. Navelet, Nucl. Phys. B 282, 727 (1987);

V.Del Duca and C.R. Schmidt, Phys. Rev. D 49, 4510 (1994);

W.J. Stirling, Nucl. Phys. B 423, 56 (1994);

R. Ragazzon and D. Treleani, Z. Phys. C 65, 305 (1995);

V.T. Kim and G.B. Pivovarov, Phys. Rev. D 53, 6 (1996); D 54, 725 (1996);

Preprint IITAP-96-12-02, Ames (1996)

[16] M.T. Grisaru, H.J. Schnitzer, and H.-S. Tsao, Phys. Rev. Lett. 30, 811 (1973);

L. Lukaszuk and X.Y. Pham, Phys. Lett. 53 B, 287 (1974);

B.M. McCoy and T.T. Wu, Phys. Rev. Lett. 35, 604 (1975); Phys. Rev. D 13, 1076 (1976);

C.Y. Lo and H. Cheng, Phys.Rev. D 13, 1131 (1976); 15, 2959 (1977);

L.L. Frankfurt and V.E. Sherman, Yad. Fiz. 23, 1099 (1976) [Sov. J. Nucl. Phys. 23, 581 (1976)];

N.T. Nieh and Y.P.Yao, Phys. Rev. D 13, 1082 (1976);

L. Tyburski, Phys. Rev. D 13, 1107 (1976);

J.B. Bronzan and R.L. Sugar, Phys.Rev. D 17, 585 (1978); 17, 2813 (1978);

H. Cheng and T.T. Wu, Expanding Protons: Scattering at High Energies (MIT Press, Cambridge, 1987)

[17] G. Sterman, An Introduction to Quantum Field Theory (Cambridge Univ., UK, 1993)

[18] V.T. Kim and G.P. Pivovarov, in progress

\section{Figure Captions}

Fig. 1. Diagrams contributing to $S_{\text {int }}$ to order $g^{2}$

Fig. 2. Divergent one-loop diagrams of the effective theory 


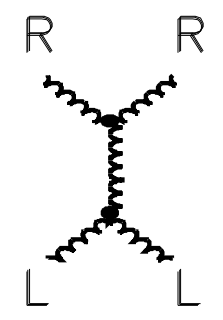

(a)

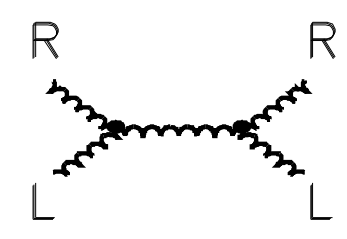

(b)

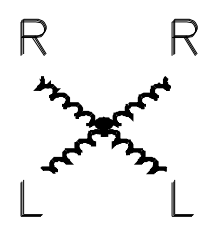

(c)

Fig. 1

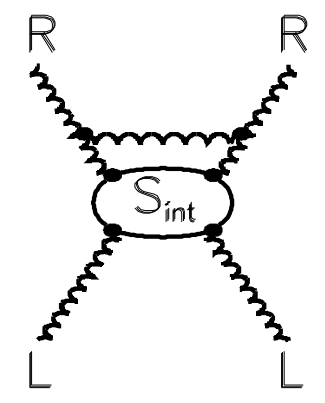

(a)

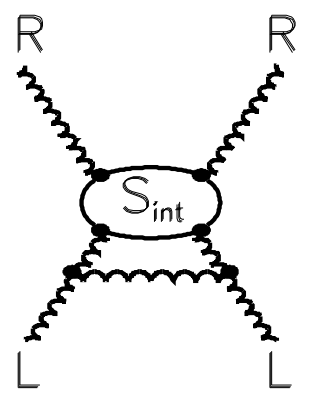

(b)

Fig. 2 